\title{
MicroRNA-301b promotes cell proliferation and apoptosis resistance in triple-negative breast cancer by targeting CYLD
}

\author{
Hongming Song, Dengfeng Li, Tianqi Wu, Dan Xie, Kaiyao Hua, Jiashu Hu, Xiaochong Deng, Changle Ji, Yijun Deng E \\ Lin Fang \\ Department of Breast and Thyroid Surgery, Shanghai Tenth People's Hospital, School of Medicine, Tongji University, Shanghai 200072, \\ China
}

\begin{abstract}
Aberrant expression of microRNAs (miRNAs) plays important roles in carcinogenesis and tumor progression. However, the expression and biological role of miR-301b in triple-negative breast cancer (TNBC) remains unclear. Here we aimed to evaluate the roles and mechanisms of miR-301b in TNBC cells. miR-301b expression was assessed in TNBC specimens and cell lines by quantitative Real-Time PCR (qRT-PCR). TNBC cells were transfected with miR-301b mimics, inhibitors or Cylindromatosis (CYLD) small interfering RNA (siRNA) using Lipofectamine 2000. The functional roles of miR-301b were determined by cell proliferation, colony formation, and apoptosis assays. Westem blots and qRT-PCR were used to measure the expression of mRNAs and proteins in the cells. We found that miR-301b was upregulated in TNBC specimens and cell lines. Overexpression of miR-301b promoted cell proliferation in TNBC cells, while inhibited the apoptosis induced by 5-FU. CYLD was downregulated by miR-301b at both mRNA and protein levels in TNBC cells. Dual-luciferase report assay confirmed that miR-301b downregulated CYLD by direct interaction with the 3 '-untranslated region(3'-UTR) of CYLD mRNA. NF-KB activation was mechanistically associated with miR-301b-mediated downregulation of CYLD. However, inhibition of miR-301b reversed all the effects of miR-301b. In conclusion, miR-301b plays an oncogenic role in TNBC possibly by downregulating CYLD and subsequently activating NF-KB p65, and this may provide a novel therapeutic approach for TNBC. [BMB Reports 2018; 51(11): 602-607]
\end{abstract}

*Corresponding author. Tel: +86-21-66307499; Fax: +86-21-6630 1869; E-mail: fanglin2017@126.com

https://doi.org/10.5483/BMBRep.2018.51.11.168

Received 22 July 2018, Revised 28 August 2018, Accepted 27 September 2018

Keywords: Apoptosis, Cell proliferation, CYLD, MiR-301b, TNBC

\section{INTRODUCTION}

TNBC is a highly aggressive subtype of breast cancers that lack estrogen receptor (ER), progesterone receptor (PR) and HER2 gene amplification, and represent $10-15 \%$ of all breast cancers (1). Patients with TNBC have a high rate of distant recurrence and a poor prognosis. Systematic chemotherapy is the primary modality used for the treatment of TNBC patients as there are no clearly defined TNBC-specific therapeutic targets. In recent years, some molecular features have been identified as biomarkers and drug targets for TNBC. The poly (ADP-ribose) polymerase (PARP) inhibitor, olaparib, could prolong the progression-free survival in a trial of BRCA-mutated breast cancer (2). miR-10b and miR-153 were significantly associated with lymph node metastases occurrence in TNBC (3). Therefore, much effort is still needed to find novel prognostic biomarkers and to clarify the molecular mechanisms underlying the development and progression of TNBC.

miRNAs are a class of highly conserved endogenous short non-coding RNAs with a length of 18-24 nucleotides. miRNAs function as negative regulators of gene expression by binding to the $3^{\prime}-$ UTR of their target mRNAs, resulting in mRNA degradation or translation suppression (4). Increasing evidence indicates that miRNAs play important roles in various biological processes, such as cell proliferation and differentiation, invasion, metastasis and apoptosis $(5,6)$. More than $50 \%$ of human miRNA genes are in cancer-associated genomic regions or in fragile sites (7). Recent studies showed that miR-301b plays important roles in pancreatic carcinoma and lung cancer $(8,9)$. However, the expression pattern and functional role of miR-301b in TNBC remain to be identified.

The NF-KB signaling pathway has key roles in inflammation, immune response, oncogenesis and protection against apoptosis $(10,11)$. Constitutive activation of NF- $\mathrm{KB}$ is often observed in breast cancer tissues and cell lines with the more aggressive phenotypes (12-15). CYLD encodes a protein with a functional deubiquitinating enzyme, which acts as a negative regulator of NF-KB signaling by deconjugating Lys63-linked polyubiquitin chains from multiple NF- $\mathrm{KB}$ regulators, such as TRAF2, TRAF6, NEMO and BCL3 (16-18). The previous study showed that reduced CYLD protein expression was

ISSN: 1976-670X (electronic edition)

Copyright (C) 2018 by the The Korean Society for Biochemistry and Molecular Biology

(c) This is an open-access article distributed under the terms of the Creative Commons Attribution Non-Commercial License (http://creativecommons.org/licenses/by-nc/4.0) which permits unrestricted non-commercial use, distribution, and reproduction in any medium, provided the original work is properly cited. 
significantly correlated with ER negativity, high Ki-67 index, decreased disease-free survival (DFS) in primary breast cancer, and CYLD downregulation was an independent factor for poor prognosis in breast cancer (19).

In this study, we demonstrated that miR-301b was upregulated in TNBC tissues and cell lines. Upregulation of miR-301b promoted cellular growth and apoptosis resistance, and inhibition of miR-301b reversed these effects in TNBC cells. Furthermore, miR-301b activated NF-KB signaling by direct targeting of the $3^{\prime}-$ UTR and suppression of CYLD in TNBC cells. The results indicate that downregulation of miR-301b might be a novel target for the diagnosis and treatment of TNBC.

\section{RESULTS}

miR-301b expression is increased in TNBC tissues and cell lines and positively correlated with Ki67 index in TNBC

To determine the clinical significance of miR-301b in the TNBC development and progression, we detected the expression of miR-301b in 38 paired of TNBC specimens and the adjacent normal breast tissues. The qRT-PCR results showed a significant increase in the expression of miR-301b in tumor tissues compared with normal tissues (Fig. 1A). Furthermore, we investigated correlations between miR-301b expression and clinicopathological factors in TNBC. The details were showed in Supplementary Table 1. Each case was

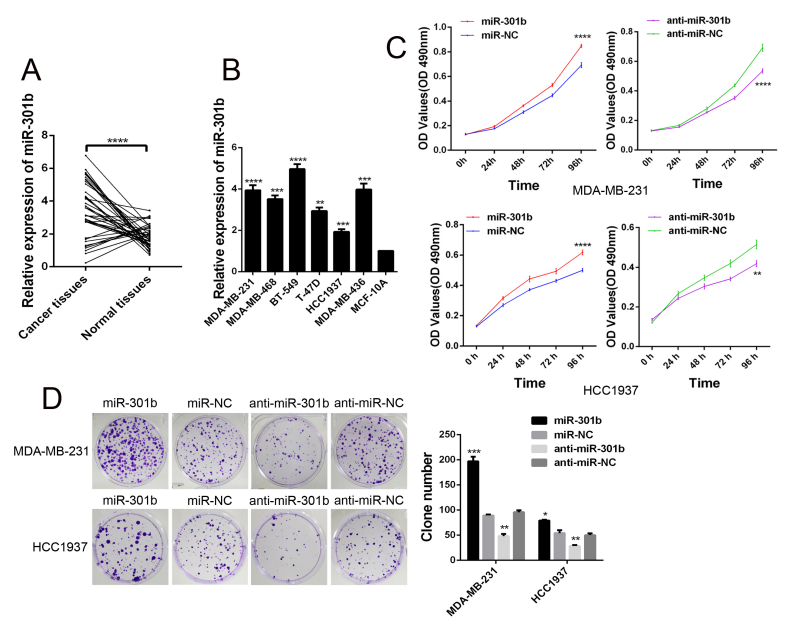

Fig. 1. miR-301b promotes the proliferation of MDA-MB-231 and HCC1937 cells. (A) The relative expression of miR-301b in 38 paired TNBC tissues and matched normal breast tissues. (B) The relative expression of miR-301b in TNBC cell lines (BT-549, MDA-MB-231, MDA-MB-468, HCC1937 and MDA-MB-436) and normal human mammary epithelial cells (MCF-10A) cells. (C, D) The proliferation was determined by MTT assay $(\mathrm{C})$ and colony formation assay (D). ${ }^{*} \mathrm{P}<0.05, * * \mathrm{P}<0.01, * * * \mathrm{P}<0.001$, $* * * * \mathrm{P}<0.0001$. TNBC, triple-negative breast cancer; MTT, 3-(4, 5-dimethyl-2-thiazolyl)-2,5-diphenyl-2H-tetrazolium bromide. subdivided into a low-expression group and a high-expression group using the mean value (1.94) of miR-301b as the cutoff value. Our results indicated that high levels of miR-301b were correlated with a high Ki-67 index (Fisher's exact test, $\mathrm{P}=$ 0.032 ) and tumor size (Fisher's exact test, $P=0.026$ ). The qRT-PCR results showed that the expression of miR-301b is significantly increased in TNBC cell lines (BT-549, MDA-MB-231, MDA-MB-468, HCC1937 and MDA-MB-436) compared with that in normal human mammary epithelial cells (MCF-10A) (Fig. 1B). The results indicated that miR-301b expression was significantly increased in TNBC tissues and cell lines, and may be associated with the development and progression of TNBC.

miR-301b promotes the proliferation of TNBC cells

To investigate the involvement of miR-301b in tumor proliferation, TNBC cell lines MDA-MB-231 and HCC1937 were transfected with the miR-301b mimics (miR-301b) or miR-301b inhibitors (anti-miR-301b), and their respective negative controls (NC). MTT assay and colony formation assays were used to evaluate the effects of miR-301b on cell proliferation. MTT results were analyzed by two-factor repeated measures ANOVA. With time-dependent, treatment with miR-NC or miR-301b showed an increased cell viability trend in MDA-MB-231 cells and HCC1937 cells. The similar results were found in the anti-miR-NC or anti-miR-301b treatment group. At the same time point, miR-301b overexpression promoted cell viability. Conversely, the cell

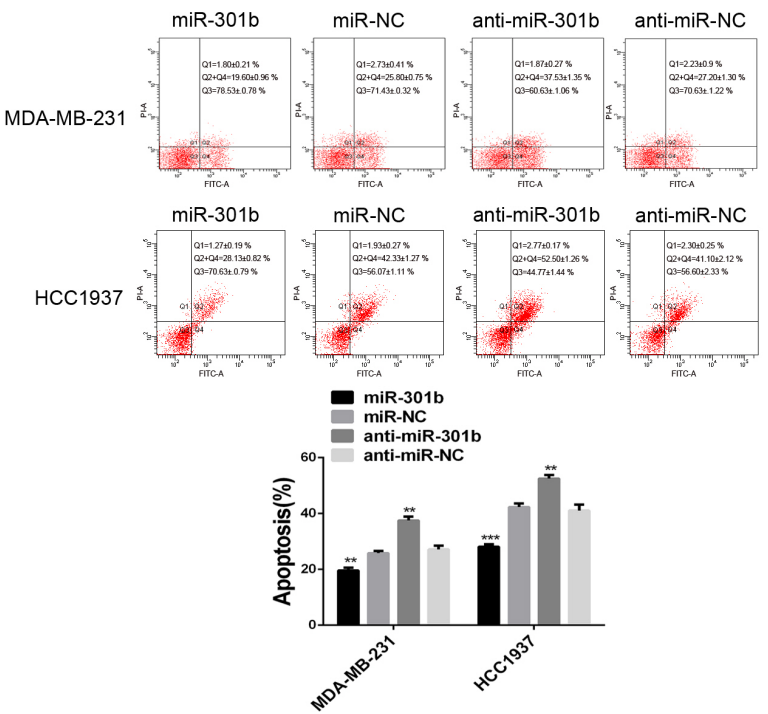

Fig. 2. miR-301b inhibits the apoptosis induced by $5-\mathrm{FU}$ in MDA-MB-231 and HCC1937 cells. The apoptosis rate was determined by Annexin V/PI assay following ectopic expression of miR-301b mimics or inhibitors in MDA-MB-231 and HCC1937 cells. Notes: quantification of proportions of Q2 + Q4 in cells represents the cell apoptosis. ${ }^{*} \mathrm{P}<0.01, * * * \mathrm{P}<0.001$. FITC, Fluorescein isothiocyanate; Pl, Propidium iodide; 5-FU, 5-fluorouraci. 
viability is suppressed in the anti-miR-301b group compared with that in anti-miR-NC (Fig. 1C). Consistently, overexpression of miR-301b increased the colony formation in both MDA-MB-231 and HCC1937 cell lines compared with the miR-NC group, while the colony formation of these cells was suppressed by inhibition of miR-301b (Fig. 1D). These results suggested that overexpression of miR-301b promoted TNBC cell proliferation, and inhibition of miR-301b reversed the effect of miR-301b on the regulation of TNBC tumorigenesis.

miR-301b inhibits apoptosis induced by 5-FU in TNBC cells Flow cytometry analysis indicated that overexpression of miR-301b increased resistance to apoptosis in 5-fluorouracil (5-FU)-treated MDA-MB-231 and HCC1937 cells compared with their respective NC cells. In contrast, inhibition of miR-301b expression could promote apoptosis of MDA-MB-231 and HCC1937 cells induced by the 5-FU treatment (Fig. 2). These results indicate that miR-301b inhibits apoptosis induced by $5-\mathrm{FU}$ in TNBC cells, while downregulation of miR-301b could increase apoptosis.

\section{miR-301b suppresses the expression of CYLD in TNBC cells,} and CYLD is a direct target of miR-301b

To confirm whether CYLD expression could be regulated by miR-301b, miR-301b, anti-miR-301b and their respective NC were transfected into TNBC cells, the results showed that the overexpression of miR-301b inhibits the expression of CYLD at mRNA and protein levels in MDA-MB-231 and HCC1937 cells, and inhibition of miR-301b results in significant upregulation of CYLD both at mRNA and protein levels (Fig. 3A, $3 B)$. Therefore, miR-301b could regulate the expression of CYLD at the mRNA and protein levels.

To determine whether CYLD is a direct target of miR-301b, specific plasmids (psiCHECK-2) containing wild-type (WT) binding sites and mutant-type (MT) binding sites of the CYLD 3'-UTR region were constructed. The predominant binding sites between miR-301b and the $3^{\prime}$-UTR of CYLD mRNA were shown in Fig. 3C-a. MDA-MB-231 cells were transfected with miR-301b or miR-NC, along with psiCHECK-2/CYLD 3'-UTR-WT or psiCHECK-2/CYLD 3'-UTR-MT. The relative luciferase activity (RL/FL) in the WT group was inhibited by miR-301b, while that in the MT group was not obviously affected by miR-301b, suggesting that miR-301b could target CYLD at the 3'-UTR (Fig. 3C-b). The above results indicated that CYLD is a direct target of miR-301b. We also used rescue experiments to confirm the regulatory relationship between miR-301b and CYLD. CYLD siRNA (si-CYLD) was used to knock down the CYLD protein expression. The expression of CYLD in the anti-miR-301b + si-CYLD groups was lower than that in the anti-miR-301b + si-NC group (Fig. 3D). These data further verify the negative regulatory between miR-301b and CYLD.

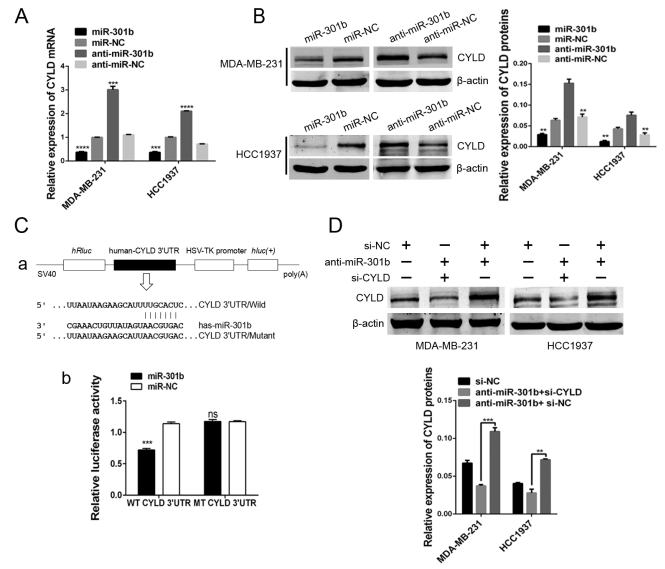

Fig. 3. miR-301b suppresses CYLD expression by directly targeting the CYLD 3 '-UTR in TNBC cells. (A) The relative expression of CYLD mRNA in MDA-MB-231 and HCC1937 cells was analyzed by qRT-PCR. The graph represents the $2^{-\Delta \mathrm{Ct}}$ values \pm SEM. (B) Western blots analysis, the relative expression of CYLD protein in MDA-MB-231 and HCC1937 cells. $\beta$-actin was used as a loading control. (C-a) Schematic of the luciferase reporter plasmids construction; (C-b) The relative luciferase activity (RL/FL) in miR-301b group was higher than that in miR-NC group after co-transfection with the CYLD 3 '-UTR-WT in MDA-MB-231 cells. But no difference was found between miR-301b group and miR-NC group after co-transfection with the CYLD 3'-UTR-MT. (D) Western blot analysis indicated that miR-301b inhibitors could rescue the downregulation of CYLD induced by CYLD siRNA at the protein level. $* * P<0.01, * * * P<0.001, * * * * P<0.0001$; qRT-PCR, quantitative Real-Time PCR; 3'-UTR, 3'-untranslated region; $R L$, renilla luciferase; $F L$, firefly luciferase; $M T$, mutant-type; $W T$, wild-type; ns, not significant.

\section{miR-301b activates the NF- $\mathrm{KB}$ signaling in TNBC cells by targeting CYLD}

Recent studies showed that NF-KB signaling contributes to the expansion of breast tumor stem cells and mammary tumorigenesis (20). We investigated the effects of miR-301b on $N F-\kappa B$ signaling in TNBC cells by analyzing the expression of NF-KB p65 subunit and the downstream genes of NF-KB. We found that miR-301b promoted expression of phosphorylated p65 (p-p65) protein, while inhibition of miR-301b decreased p-p65 protein expression. There had no effect on the total p65 protein expression by overexpression and downregulation of miR-301b (Fig. 4A, 4B). The qRT-PCR results showed that the expression of NF-KB downstream genes, such as Bcl-2, XIAP, and IL-8, were significantly higher in the miR-301b group than in the miR-NC group, and inhibition of miR-301b could decrease the mRNA expression of Bcl-2, XIAP, and IL-8 (Fig. $4 C)$. In addition, knockdown of CYLD by siRNA increased the expression of p-p65 protein, but did not alter the total p65 protein in HCC1937 cells (Fig. 4D), as well as increased the NF-KB-driven gene transcription, including XIAP, Bcl-2, and 


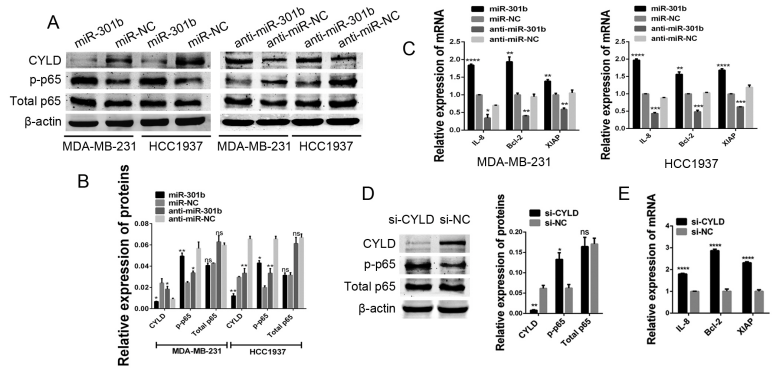

Fig. 4. miR-301b activates the NF- $\mathrm{kB}$ signaling in TNBC cells by targeting CYLD. (A, B) Expression of CYLD, total p65, and p-p65 protein were analyzed by western blot after transfection with miR-301b and anti-miR-301b. $\beta$-actin served as a loading marker. (C) The expression of XIAP, BCl-2, and IL-8 at the mRNA level were analyzed by qRT-PCR after transfection with miR-301b and anti-miR-301b. (D, E) The protein expression of CYLD, total p65 and p-p65 (D), and the mRNA expression levels of XIAP, BCl-2, and IL-8 (E) were measured after downregulation of CYLD by siRNA in HCC1937 cells. $\beta$-actin served as a loading control. *P $<0.05,{ }^{* * P}<0.01,{ }^{* * * P}<0.001,{ }^{* * * * P}<0.0001 ; \mathrm{ns}$, not significant; siRNA, small interfering RNA.

IL-8 (Fig. 4E). All these results showed that miR-301b targets the NF-KB pathway by suppressing the expression CYLD and activation of NF-kB p65.

\section{DISCUSSION}

Deregulated expression of miRNAs is associated with tumor progression in various types of cancers. In this study, we found that miR-301b expression was upregulated in TNBC tissues and cell lines when compared with normal tissues and cell lines, and high levels of miR-301b were correlated with tumor size and high Ki-67 index in TNBC samples. The expression of Ki67 correlated strongly with tumor cell proliferation and growth and is widely used as a prognostic and predictive marker for breast cancer diagnosis and treatment. This means that miR-301b may be associated with the development and progression of TNBC. We found over-expression of miR-301b significantly increased the proliferation and suppressed the apoptosis induced by 5-FU in MDA-MB-231 and HCC1937 cells, and the effects were reversed by inhibition of miR-301b. These results suggest that miR-301b functions as an oncogene in TNBC. miR-301b is upregulated in several cancers, such as pancreatic, colorectal and lung carcinoma $(8,9,21)$. TP63 and Bim have been confirmed to be the target genes of miR-301b $(8,9)$. For example, miR-301b promotes cell invasiveness by targeting TP63 in pancreatic carcinoma cells. The luciferase reporter assay suggested that CYLD was an important target of miR-301b in TNBC cells. Loss of CYLD is associated with tumorigenesis (22). The reduced CYLD expression has been detected in several malignancies, such as hepatocellular carcinoma, breast cancer $(19,23)$. The expression of CYLD gene is decreased in breast cancer tissues and cells (19), which means that there is a potential association of CYLD gene suppression with breast cancer. However, why CYLD expression is suppressed in breast cancer tissues and cells has not been fully elucidated. The results from BT-549 cells, a TNBC cell line, suggest that the CYLD expression is directly regulated by BAF57, a key hSWI/SNF subunit that regulates gene expression through chromatin remodeling (24). We identified CYLD gene as a target for miR-301b, high expression of miR-301b may be one of the reasons for CYLD gene repression in TNBC. Knockdown of CYLD by specific siRNA causes increased cell viability, with decreased apoptosis in MDA-MB-231 cells (19), which indicated that miR-301b plays an oncogenic role in TNBC cells at least partly by targeting CYLD. In addition, the expression of CYLD gene in melanoma cells is suppressed by the zinc finger transcription factor, Snail, which is involved in the proliferation and invasion of melanoma cells (25).

CYLD was identified as a negative regulator for NF-kB signaling (17). One study found that CYLD binds to the NF-KB essential modulator (NEMO), which is a component of the IKB kinase (IKK) complex. Activation of IKK phosphorylates NF-KB inhibitory proteins $(\mathrm{I} \mathrm{KBS})$, thereby triggering the degradation of IKBs and the activation of NF-KB. CYLD knockdown or knockout promotes ubiquitination of NEMO and several IKK upstream regulators such as Tak1, TRAF2, TRAF6, leading to activation of the NF- $\mathrm{kB}$ signaling pathway (22). For patients with TNBC, the 5-year DFS and breast cancer-specific survival rates in patients with CYLD-negative tumors were less than those in patients with CYLD-positive tumors (19). Inhibition of constitutive NF-KB activation suppressed proliferation of the breast cancer cell lines HCC1937 and MDA-MB-231 cells (12). We found that overexpression of miR-301b significantly increased the expression of NF-kB p-p65, while inhibition of miR-301b reduced the expression of NF- $\mathrm{kB}$ p-p65 in MDAMB-231 and HCC1937 cells. In addition, CYLD knockdown significantly increased NF-KB activity and the expression levels of the NF-kB pathway-associated genes in these cells. These results demonstrate that miR-301b may activate the NF- $\mathrm{KB}$ signaling pathway by inhibiting CYLD.

In summary, our study has demonstrated that miR-301b was upregulated in TNBC tissues and cell lines, and positively correlated with Ki67 index in TNBC. It was able to induce cell proliferation and apoptosis resistance by negatively regulating the expression of CYLD in TNBC cells. miR-301b plays an oncogenic role in TNBC cells by CYLD-mediated NF- $\mathrm{KB}$ activation. This study suggests that miR-301b may serve as a novel biomarker and the miR-301b-CYLD axis may be a potential therapeutic target for TNBC patients.

\section{MATERIALS AND METHODS}

\section{Specimens}

The 38 pairs of TNBC samples and their matched normal adjacent tissues were collected at the Department of Breast 
and Thyroid Surgery of Shanghai Tenth People's Hospital, Shanghai, China. All patients participating in this study had signed the informed consent prior to their inclusion in the study. The experimental research and the collection of patient's tissues for the study was approved by the Ethics Committee of the Hospital (reference number: SHSY-IECKY-4.0/17-83/01).

\section{Cell culture and transfection}

TNBC cell lines BT-549, MDA-MB-231, MDA-MB-468, HCC1937 and MDA-MB-436 were obtained from the cell bank of the Chinese Academy of Science (Shanghai, China). Human normal breast cell line MCF-10A was purchased from Zhongqiaoxinzhou Biotech (Shanghai, China). BT-549 cells were cultured in RPMI 1640 (Gibco, USA) supplemented with $10 \%$ fetal bovine serum (FBS, Gibco, USA), 1\% penicillinstreptomycin (PS, $100 \mu \mathrm{g} / \mathrm{ml}$ ) (Enpromise, Hangzhou,China). MCF10A cells were cultured in Mammary Epithelial Cell Medium (MEpiCM, ScienCell, USA). The other cell lines were cultured in DMEM medium (Gibco, USA) containing 10\% FBS, $1 \%$ PS. All of the cell lines were grown in an incubator with humidified air $\left(5 \% \mathrm{CO}_{2}\right)$ at $37^{\circ} \mathrm{C}$.

At $24 \mathrm{~h}$ prior to transfection, cells were plated into 6-well plates at a density of $1.0 \times 10^{5}$ cells per well. When the cells reached 30-40\% confluence, miR-301b/miR-NC, anti-miR-301b/ anti-miR-NC (RiboBio, China), and si-CYLD/si-NC (Sangon Biotech, China) were transiently transfected at a final concentration of $100 \mathrm{nmol} / \mathrm{l}$ in MDA-MB-231 and HCC1937 cells using Lipofectamine 2000 (Invitrogen, USA). In the rescue experiment, HCC1937 cells were transiently cotransfected with $100 \mathrm{nmol} / \mathrm{l}$ siRNA (si-CYLD or si-NC) and 100 nmol/I anti-miR-301b with Lipofectamine 2000 (Invitrogen, USA).

\section{Protein extraction and western blot analysis}

Total protein extraction and western blot analysis were performed according to standard methods as described previously (26). Primary antibodies for western blot are listed as follows:polyclonal anti-rabbit CYLD antibody (1:1000, Proteintech, China), anti-rabbit NF-KB p65 (1:1000, Cell Signaling, USA), anti-rabbit Phospho-NF-кB p65 (Ser536) (1:500, Cell Signaling, USA) as well as corresponding secondary anti-rabbit or anti-mouse antibodies. The western blot results were analyzed by Odyssey infrared imaging software.

\section{RNA Extraction and $q R T-P C R$}

Total RNA extraction and qRT-PCR were performed as previously described (26). Expression of mRNAs or miRNAs were assessed by evaluating threshold cycle $(\mathrm{Ct})$ values. $\beta$-actin mRNA was employed as an endogenous control for mRNAs. U6 RNA was used to normalize miRNAs. The relative fold difference was calculated using the $2^{-\Delta \Delta \mathrm{Ct}}$ formula. The primers for PCR were designed by Sangon Biotech (Shanghai,
China) and the primer sequences were shown in Supplementary-Table-2.

\section{Cell viability assay}

The MDA-MB-231 and HCC1937 cells were seeded with complete medium in 96-well plates at a density of 500 cells/well in a total volume of $200 \mathrm{ul}$. $20 \mu \mathrm{l}$ 3-(4, 5-dimethyl2-thiazolyl)-2,5-diphenyl-2H-tetrazolium bromide (MTT) solution ( $5 \mathrm{mg} / \mathrm{ml}$, Sigma-Aldrich, USA) was added into each well and the plate was incubated for 4 hours in $37^{\circ} \mathrm{C}$. The reaction was terminated with $150 \mu \mathrm{l}$ of dimethylsulfoxide (DMSO, SigmaAldrich, USA) per well. The plate was then read for absorption at the optical density (OD) of $490 \mathrm{~nm}$ on a microplate reader. Five replicates of each treatment were used, and the experiment was performed three times.

\section{Colony formation assay}

The transfected cells (500 cells/well) were plated in 6-well plates containing complete medium and cultured for 7-10 days. The colonies were subsequently fixed in $10 \%$ formaldehyde for $10 \mathrm{~min}$ and stained with $1.0 \%$ crystal violet for $20 \mathrm{~min}$. Cell colonies with diameters more than $1.5 \mathrm{~mm}$ were counted. The experiment was repeated three times.

\section{Apoptosis assay}

MDA-MB-231 and HCC1937 cells were transfected with miR-301b and anti-miR-301b, and followed by treatment with 5-FU $(10 \mathrm{mM})$ for $36 \mathrm{~h}$ to induce the apoptosis. Apoptosis was detected by the FITC Annexin V Apoptosis Detection Kit (BD Biosciences) following the manufacturer's instructions. The stained cells were analyzed by FACS Calibur flow cytometry. The percentage of apoptotic cells was quantified by the sum of Annexin $\mathrm{V}+/ \mathrm{PI}-$ and Annexin $\mathrm{V}+/ \mathrm{PI}+$ cells, as shown in the Q2 and Q4 quadrants of the FACS assay.

\section{Luciferase reporter assay}

To prove direct targeting by miR-301b, psiCHECK2 vectors (40 ng, IBS-Bio, China), containing the respective wild-type or mutated human CYLD 3-'UTR, were co-transfected with miR-301b or miR-NC (100 nmol/l) in MDA-MB-231 cells. Renilla and firefly luciferase activities were measured $24 \mathrm{~h}$ post-transfection. Renilla luciferase (RL) values were normalized to firefly luciferase (FL) values and expressed as fold change compared to control cells.

\section{Statistical analysis}

Statistical analyses were performed with GraphPad Prism version 6.0 (GraphPad, USA) or SPSS 20.0 (IBM, USA). All data are presented as the mean \pm standard error of the mean (SEM) from three independent assays. We utilized the Fisher's exact for statistical analysis of the associations between miR-301b expression and clinicopathological factors. Twofactor repeated measures ANOVA were used for analysis of MTT results. The Student's t-test was used for the comparison 
of two means. A P-value less than 0.05 was considered statistically significant.

\section{ACKNOWLEDGEMENTS}

This work was supported by grants from the National Natural Science Foundation of China (No: 82172240), and the Shanghai Municipal Health Bureau of Shanghai, China (No: 201640097).

\section{CONFLICTS OF INTEREST}

The authors have no conflicting interests.

\section{REFERENCES}

1. Dawood S (2010) Triple-negative breast cancer: epidemiology and management options. Drugs 70, 2247-2258

2. Lee A and Djamgoz MBA (2018) Triple negative breast cancer: Emerging therapeutic modalities and novel combination therapies. Cancer Treat Rev 62, 110-122

3. Fkih M'hamed I, Privat M, Trimeche M, Penault-Llorca F, Bignon YJ and Kenani A (2017) miR-10b, miR-26a, miR-146a and miR-153 expression in triple negative vs non triple negative breast cancer: Potential biomarkers. Pathol Oncol Res 23, 815-827

4. Griffiths-Jones S, Grocock RJ, van Dongen S, Bateman A and Enright AJ (2006) miRBase: microRNA sequences, targets and gene nomenclature. Nucleic Acids Res 34, D140-144

5. Ardekani AM and Naeini MM (2010) The role of microRNAs in human diseases. Avicenna J Med Biotechnol 2, 161-179

6. Tufekci KU, Meuwissen RL and Genc S (2014) The role of microRNAs in biological processes. Methods Mol Biol 1107, 15-31

7. Calin GA, Sevignani C, Dumitru CD et al (2004) Human microRNA genes are frequently located at fragile sites and genomic regions involved in cancers. Proc Natl Acad Sci U S A 101, 2999-3004

8. Wu D, Chen B, Cui F, He X, Wang W and Wang M (2016) Hypoxia-induced microRNA-301b regulates apoptosis by targeting Bim in lung cancer. Cell Prolif 49, 476-483

9. Funamizu N, Lacy CR, Parpart ST, Takai A, Hiyoshi $Y$ and Yanaga K (2014) MicroRNA-301b promotes cell invasiveness through targeting TP63 in pancreatic carcinoma cells. Int J Oncol 44, 725-734

10. Karin M and Ben-Neriah Y (2000) Phosphorylation meets ubiquitination: the control of NF-[kappa]B activity. Annu Rev Immunol 18, 621-663

11. Li Q and Verma IM (2002) NF-kappaB regulation in the immune system. Nat Rev Immunol 2, 725-734

12. Yamaguchi N, Ito T, Azuma $S$ et al (2009) Constitutive activation of nuclear factor-kappaB is preferentially involved in the proliferation of basal-like subtype breast cancer cell lines. Cancer Sci 100, 1668-1674

13. Biswas DK, Shi Q, Baily S et al (2004) NF-kappa B activation in human breast cancer specimens and its role in cell proliferation and apoptosis. Proc Natl Acad Sci U S A 101, 10137-10142

14. Sovak MA, Bellas RE, Kim DW et al (1997) Aberrant nuclear factor-kappaB/Rel expression and the pathogenesis of breast cancer. J Clin Invest 100, 2952-2960

15. Zhou Y, Eppenberger-Castori S, Marx C et al (2005) Activation of nuclear factor-kappaB (NFkappaB) identifies a high-risk subset of hormone-dependent breast cancers. Int J Biochem Cell Biol 37, 1130-1144

16. Kovalenko A, Chable-Bessia C, Cantarella G, Israel A, Wallach D and Courtois G (2003) The tumour suppressor CYLD negatively regulates NF-kappaB signalling by deubiquitination. Nature 424, 801-805

17. Brummelkamp TR, Nijman SM, Dirac AM and Bernards R (2003) Loss of the cylindromatosis tumour suppressor inhibits apoptosis by activating NF-kappaB. Nature 424, 797-801

18. Trompouki E, Hatzivassiliou E, Tsichritzis $T$, Farmer $H_{\text {, }}$ Ashworth A and Mosialos G (2003) CYLD is a deubiquitinating enzyme that negatively regulates NF-kappaB activation by TNFR family members. Nature 424, 793-796

19. Hayashi $M$, Jono $H$, Shinriki $S$ et al (2014) Clinical significance of CYLD downregulation in breast cancer. Breast Cancer Res Treat 143, 447-457

20. Liu M, Sakamaki T, Casimiro MC et al (2010) The canonical NF-kappaB pathway governs mammary tumorigenesis in transgenic mice and tumor stem cell expansion. Cancer Res 70, 10464-10473

21. Wang YX, Zhang XY, Zhang BF, Yang CQ, Chen XM and Gao HJ (2010) Initial study of microRNA expression profiles of colonic cancer without lymph node metastasis. J Dig Dis 11, 50-54

22. Sun SC (2010) CYLD: a tumor suppressor deubiquitinase regulating NF-kappaB activation and diverse biological processes. Cell Death Differ 17, 25-34

23. Hellerbrand C, Bumes E, Bataille F, Dietmaier W, Massoumi R and Bosserhoff AK (2007) Reduced expression of CYLD in human colon and hepatocellular carcinomas. Carcinogenesis 28, 21-27

24. Wang L, Baiocchi RA, Pal S, Mosialos G, Caligiuri M and Sif $S$ (2005) The BRG1- and hBRM-associated factor BAF57 induces apoptosis by stimulating expression of the cylindromatosis tumor suppressor gene. Mol Cell Biol 25, 7953-7965

25. Massoumi R, Kuphal S, Hellerbrand C et al (2009) Down-regulation of CYLD expression by Snail promotes tumor progression in malignant melanoma. J Exp Med 206, 221-232

26. Song HM, Song JL, Li DF, Hua KY, Zhao BK and Fang L (2015) Inhibition of FOXO1 by small interfering RNA enhances proliferation and inhibits apoptosis of papillary thyroid carcinoma cells via Akt/FOXO1/Bim pathway. Onco Targets Ther 8, 3565-3573 